\title{
Relación entre la práctica de actividad física y los empleados saludables en un club deportivo-social
}

\section{Relationship between practicing physical activity and healthy employees in a sports-social club}

\section{Relação entre prática de atividade física e funcionários saudáveis em um clube social-esportivo}

\author{
Gómez-Chacón, R.; Fernández-Martínez, N. \\ CEU Cardenal Spínola CEU
}

\begin{abstract}
RESUMEN
En la actualidad las organizaciones están implementando acciones de gestión de la salud por medio de la actividad física repercutiendo en beneficios físicos, psicológicos y sociales en los empleados y en económicos en las empresas. Este hecho hace que las organizaciones dispongan de empleados saludables que se relacionan con variables organizacionales positivas, como la satisfacción laboral, por lo que el objetivo del trabajo es conocer la relación entre la práctica de actividad física y los empleados saludables. Los participantes fueron los empleados de una entidad deportivo-social de la provincia de Sevilla con $n=56$, de los cuales 29 hombres y 27 mujeres, además se utilizó el cuestionario internacional de actividad física (IPAQ) y el cuestionario del empleado saludable, analizando si existían diferencias significativas entre las variables del empleado saludable y la cantidad de práctica de actividad física de los empleados. Los resultados concluyen que los empleados que realizan actividad física moderada y alta presentan un promedio significativamente mayor en emociones positivas, engagement y resiliencia respecto al grupo de actividad física baja o no práctica de actividad física, mientras que los empleados que realizan actividad física moderada y alta presentan un promedio mayor, pero no significativo en autoeficacia y competencia respecto al grupo de actividad física baja o no práctica de actividad física.
\end{abstract}

Palabras clave: Actividad física, organizaciones, empleados.

\begin{abstract}
Organisations are currently implementing health management actions through physical activity, impacting physical, psychological and social benefits for employees and economic benefits for companies. This fact makes organizations have healthy employees that are related to positive organizational variables, such as job satisfaction, so the objective of the work is to know the relationship between the practice of physical activity and healthy employees. Participants were employees of a sports-social entity in the province of Seville with $\mathrm{n}=56$, of whom 29 were men and 27 women, and the International Physical Activity Questionnaire (IPAQ) and the Healthy Employees Questionnaire were also used. analyzing if there were significant differences between the variables of the healthy employee and the amount of physical activity practice of the employees. The results conclude that the employees who carry out moderate and high physical activity present a significantly higher average in positive emotions, commitment and resistance compared to the group of low physical activity or who do not practice physical activity, while employees who carry
\end{abstract}

Correspondence to: Ramón Gómez Chacón. Calle San Fernando, 4, 41004 Sevilla. Email: rgomez@ceuandalucia.es 


\section{Gómez-Chacón, R; Fernández-Martínez, N.}

out moderate physical activity and high have a higher average, but not significant in self-efficacy and competence with respect to the group of low physical activity or that does not practice physical activity.

Keywords: Physical activity, organisations, employees.

\section{RESUMO}

Atualmente, as organizações estão implementando ações de gestão em saúde por meio de atividade física, impactando benefícios físicos, psicológicos e sociais para os funcionários e benefícios econômicos para as empresas. Esse fato faz com que as organizações tenham funcionários saudáveis relacionados a variáveis organizacionais positivas, como satisfação no trabalho, de modo que o objetivo do trabalho é conhecer a relação entre a prática de atividade física e funcionários saudáveis. Os participantes eram funcionários de uma entidade social-esportiva da província de Sevilha com $\mathrm{n}=56$, dos quais 29 eram homens e 27 mulheres, e também foram utilizados o Questionário Internacional de Atividade Física (IPAQ) e o Questionário de Funcionários Saudáveis. analisando se havia diferenças significativas entre as variáveis do empregado saudável e a quantidade de prática de atividade física dos empregados. Os resultados concluem que os empregados que realizam atividade física moderada e alta apresentam uma média significativamente maior de emoções positivas, comprometimento e resistência em comparação ao grupo de baixa atividade física ou que não praticam atividade física, enquanto empregados que realizam atividade física moderada e alta têm uma média mais alta, mas não significativa em autoeficácia e competência em relação ao grupo de baixa atividade física ou que não pratica atividade física.

Palavras chave: Atividade física, organizações, funcionários.

\section{INTRODUCCIÓN}

Las organizaciones españolas están iniciándose desde hace algunos años en el diseño e implementación de acciones de gestión y promoción de la salud por medio de la actividad física. Este hecho permite que sean además de un lugar de trabajo, un entorno de promoción de la actividad física encaminada a mejorar la productividad y mejorar la salud, por sus grandes beneficios físicos y psicológicos (Netz, Wu, Becker y Tenenbaum, 2005). Además, ha propiciado que las organizaciones y sus dirigentes se preocupen por la salud de sus empleados naciendo el concepto de organizaciones saludables, que se definen como aquellas organizaciones que realizan esfuerzos sistemáticos, planificados y proactivos para mejorar la salud de los empleados mediante buenas prácticas relacionadas con la mejora de las tareas (por ejemplo, con el diseño y rediseño de puestos), el ambiente social (por ejemplo con mejores canales de comunicación abierta) y la organización (estrategias de conciliación trabajo/vida privada) (Salanova, 2008; Salanova y Schaufeli, 2009). Los empleados saludables son los que integran estas organizaciones saludables y cuentan con fortalezas y capacidades psicológicas que pueden ser medidas, desarrolladas y gestionadas para conseguir la mejora del funcionamiento organizacional y el desempeño (Salanova, 2008). Y se identifican por ciertas características o fortalezas personales como son la autoeficacia, la esperanza, el optimismo y la resiliencia (Luthans y Youssef, 2004; Stanjovik, 2006), además del engagement (Salanova, 2008; Salanova, 2009). Las diferentes fortalezas del empleado saludable se relacionan positivamente con variables organizacionales y con variables que inciden en la salud de los empleados, como por el ejemplo el engagement positivamente con el liderazgo (Bamford, Wong y Laschinger, 2013), con el absentismo laboral (Palací, 2009), el desempeño (Lisbona, Morales y Palací, 2009), el contagio emocional (Acosta, Salanova y Llorens, 2011). La autoeficacia se relaciona positivamente con la motivación (Vera, Le Blanc, Taris y Salanova, 2014), desempeño y bienestar (Salanova, Martínez y Llorens, 2012). La resiliencia se relaciona positivamente con la productividad y el rendimiento (Meneghel, Salanova y Martínez, 2013). El optimismo se relaciona positivamente con la motivación y creatividad (Isen, 2002). Y la esperanza se relaciona positivamente con la salud, la satisfacción laboral y el rendimiento (Nelson y Simmons, 2006).

Por otro lado, la práctica de actividad física en el ámbito laboral ha ido progresivamente aumentando en las organizaciones debido en parte a la estrategia NAOS, que comenzó en el año 2005, y la cual desarrolla acciones o intervenciones, en diferentes ámbitos de la sociedad (familiar, educativo, empresarial, sanitario, laboral, comunitario) 


\section{Actividad física y empleados saludables}

(Ministerio de Sanidad y Consumo, 2005). En este sentido la práctica de actividad física se relaciona positivamente con, la productividad laboral (De Miguel, Schweiger, De las Mozas y Hernández, 2011), percepción estado de salud y salud en general (Casimiro, Artés, Muyor y Rodriguez, 2011), entusiasmo y bienestar psicológico (ThøgersenNtoumani, Fox, y Ntoumanis, 2005), confianza (Díaz, Mena y Rebolledo, 2012), y satisfacción laboral (Gómez, Grimaldi, Bernal y Fernández, 2016; Gómez, Morales, Hernández y Muñoz, 2018), estabilidad emocional y optimismo (Boix, León y Serrano, 2017), mejores parámetros psicofisiológicos y sociales relacionados con dolores musculoesqueléticos, sensación de bienestar y relaciones interpersonales (Magno et al., 2019; Silva et al., 2019), Índice de masa corporal, el peso corporal, la masa grasa, colesterol, física fi aptitud y la calidad del sueño (Flahr, Brown y Kolbe-Alexander, 2018), capacidad de control del peso (Täuber, Mulder, y Flint, 2018).

Por otro lado, Gómez-Chacón (2017) demostró que en una muestra de tres empresas de diferentes sectores como la alimentación, informática y consultoría, las cinco variables del empleado saludable muestran índices significativamente mayores en los trabajadores que practican actividad física alta y moderada en relación con los no practicantes de actividad física.

Debido a los pocos estudios que relacionan la práctica de actividad física con empleados saludables en las organizaciones, el objetivo principal de este estudio es conocer la relación entre la práctica de actividad física y los empleados saludables y sus fortalezas. Este trabajo aporta el análisis de las fortalezas del empleado saludable relacionándolas con la práctica de actividad física, un hecho que se está estudiando en la actualidad y del que se disponen pocos estudios.

\section{MATERIAL Y MÉTODOS}

\section{Participantes.}

Los participantes son los empleados de un centro deportivo-social de un municipio de la provincia de Sevilla, que tienen 65 empleados, obteniendo con un margen de error del 5 y nivel de confianza del $95 \%, n$ $=56$. De los cuales 29 hombres y 27 mujeres entre 18 y 60 años, encontrándose 18 entre 18-30 años, 30 entre 30-45 años y 8 entre 45 y 60 años (Tabla 1 ).
La particularidad del centro es que una amplia mayoría trabaja en jornada parcial con $n=35$, mientras que con jornada completa $n=21$. Además, el $50 \%$ de los empleados que participaron en el estudio eran técnicos deportivos, mientras que el otro $50 \%$ pertenecían a servicios general y restauración.

Tabla 1. Sexo y edad.

\begin{tabular}{ccccccc}
\hline & \multicolumn{9}{c}{ Edad } & & & \\
& $18-30$ & $30-45$ & $45-60$ & M & D.T & Total \\
\hline \multirow{2}{*}{ Sexo hombre } & 9 & 18 & 2 & 38.7 & .577 & 29 \\
mujer & 9 & 12 & 6 & 33.4 & .751 & 27 \\
Total & 18 & 30 & 8 & 36.05 & .664 & 56 \\
\hline
\end{tabular}

\section{Instrumentos.}

Para valorar la práctica de actividad física se utilizó el IPAQ que tiene aceptables propiedades de medición para usarse en diferentes lugares y en diferentes idiomas, y también que es aceptable y apropiado para investigaciones nacionales de participación en actividad física (Brown, Trost, Bauman, Mummery y Owen, 2004; Pardini y Matsudo, 2001). Diversos autores han llevado a cabo estudios en los cuales se ha estudiado las características psicométricas del IPAQ y confirmado su validez con una fiabilidad medida a través del alfa de Cronbach de 0.88 para la versión corta en español (Brown et al., 2004; Craig et al., 2003). Por otro lado, se clasificaron a los empleados en tres categorías, alta(1), media(2), y baja o no práctica de actividad física(3) según la actividad física desarrollada.

Además, se utilizó el cuestionario del empleado saludable (Salanova et al., 2012) en su versión reducida de ocho a cinco dimensiones llevada a cabo por Gómez-Chacón, García-Fernández, MoralesSánchez y Hernández-Mendo (2020), donde se reduce de ocho dimensiones tales como, engagement vigor, engagement dedicación y engagement absorción, autoeficacia, resiliencia, optimismo, competencia mental y competencia emocional, a cinco dimensiones, tales como engagement, competencia, autoeficacia, resiliencia y emociones positivas. En ambos modelos, de ocho dimensiones y cinco dimensiones, muestran un buen ajuste, ya que los índices incrementales (CFI y NNFI) y el absoluto SRMR son superiores a .90 e inferior a .08 respectivamente (Gómez-Chacón, 2017). Mostrando 


\section{Gómez-Chacón, R; Fernández-Martínez, N.}

una buena consistencia interna en el modelo de cinco dimensiones, obteniendo valores superiores a .80 en todas sus escalas, de modo que en emociones positivas $=.87$, en engagement $=.92$, en resiliencia $=.83$, en competencia $=.88$, y en autoeficacia $=.87$ (GómezChacón, García-Fernández, Morales-Sánchez y Hernández-Mendo, 2020). El cuestionario consta de 40 preguntas siendo las seis primeras para medir el optimismo por medio de las emociones positivas con una escala tipo Likert de 7 puntos, donde 0 es nada y 6 es siempre. Las 18 siguientes miden el engagement con una escala tipo Likert de 7 puntos, donde 0 es nada y 6 es siempre. Las siete siguientes miden la resiliencia con una escala tipo Likert de 7 puntos, donde 0 es nada y 6 es siempre. Las tres siguientes miden la autoeficacia con una escala tipo Likert de 7 puntos, donde 0 es incapaces de hacerlo y 6 es seguro de hacerlo bien. Y las tres últimas miden la esperanza por medio de la competencia con una escala tipo Likert de 7 puntos, donde 0 es nada y 6 es siempre.

\section{Diseño de investigación.}

Se realizó un estudio cuantitativo descriptivo y de corte transversal. La información se obtuvo por medio de una muestra conveniente y no probabilística.

El estudio respeta los principios éticos de las investigaciones científicas y se han seguido los requisitos establecidos por la American Phychological Association (American Psycological Association, 2002).

\section{Procedimiento.}

Se llevo a cabo una entrevista con el gerente del centro deportivo informando del proceso y los beneficios de llevar a cabo el estudio, en ese momento se diseñó un cuestionario en formato Google forms que se envió al gerente del centro deportivo, siendo éste el que enviara el enlace online a sus empleados. Las respuestas de los empleados se llevaron a cabo entre 21-28 de enero de 2019 y se recopilaron en un documento Excel.

\section{Análisis de datos.}

Se realizó la prueba de normalidad entre las variables del empleado saludable de la muestra respecto a los empleados que practican actividad física alta, moderada y baja. El cumplimiento nos lleva a realizar pruebas paramétricas ómnibus F de ANOVA en todas las dimensiones, excepto con las emociones positivas y actividad física alta y la autoeficacia y actividad física baja que se llevó a cabo pruebas no paramétricas ANOVA kruskal-wallis, estas pruebas se realizaron para comprobar si existen diferencias significativas en los sumatorios de las escalas del empleado saludable entre los tres niveles de práctica de actividad física.

Por último, se llevarán a cabo las pruebas post hoc que se realizan para concretar y detallar si existen diferencias significativas entre los diferentes grupos, es decir entre los tipos de actividad física; actividad física vigorosa, actividad física moderada y actividad física baja. En este caso se utiliza la corrección del $\alpha$ por Bonferroni, el método más utilizado y conocido, aunque más conservador.

Se llevaron a cabo todos los análisis con el programa estadístico SPSS 24.

\section{RESULTADOS}

El análisis descriptivo de los diferentes indicadores del empleado saludable con respecto a los tres niveles de actividad indica en primer lugar que en emociones positivas y actividad física alta el ítem 3 y 4 tienen la mayor puntuación con $M=5,14$ y emociones positivas y actividad física baja el ítem 1 la menor puntuación con $M=2,13$.

Con respecto al engagement y la de actividad física alta el ítem 9 tiene la puntuación más alta $M=5,67$, y la puntuación más baja se encuentra en engagement con actividad física baja el ítem $2 \operatorname{con} M=2,75$.

La resiliencia y la actividad física alta tienen en su ítem 2 con $M=5,19$ su puntuación más alta, y resiliencia y actividad física baja en su ítem $3 \operatorname{con} M=$ 3,00 su puntuación más baja.

La competencia y actividad física alta tiene la puntuación más alta en el ítem 1 con $M=4,95$, y la puntuación menor en resiliencia y actividad física baja en el ítem 3 con $M=3,81$.

Y la autoeficacia y actividad física alta tiene su puntuación más alta en el ítem 1 con $M=5,14$, y la más baja en autoeficacia y actividad física moderada en el ítem 3 con $M=4,58$. 


\section{Actividad física y empleados saludables}

En la Tabla 2 se muestra el supuesto de normalidad de todas las dimensiones del empleado saludable junto con la actividad física alta, moderada y baja, siendo todas $p>, 05$, excepto en engagement y actividad física alta, y autoeficacia y actividad física baja con $p$ $<, 05$.

Tabla 2. Supuesto de normalidad.

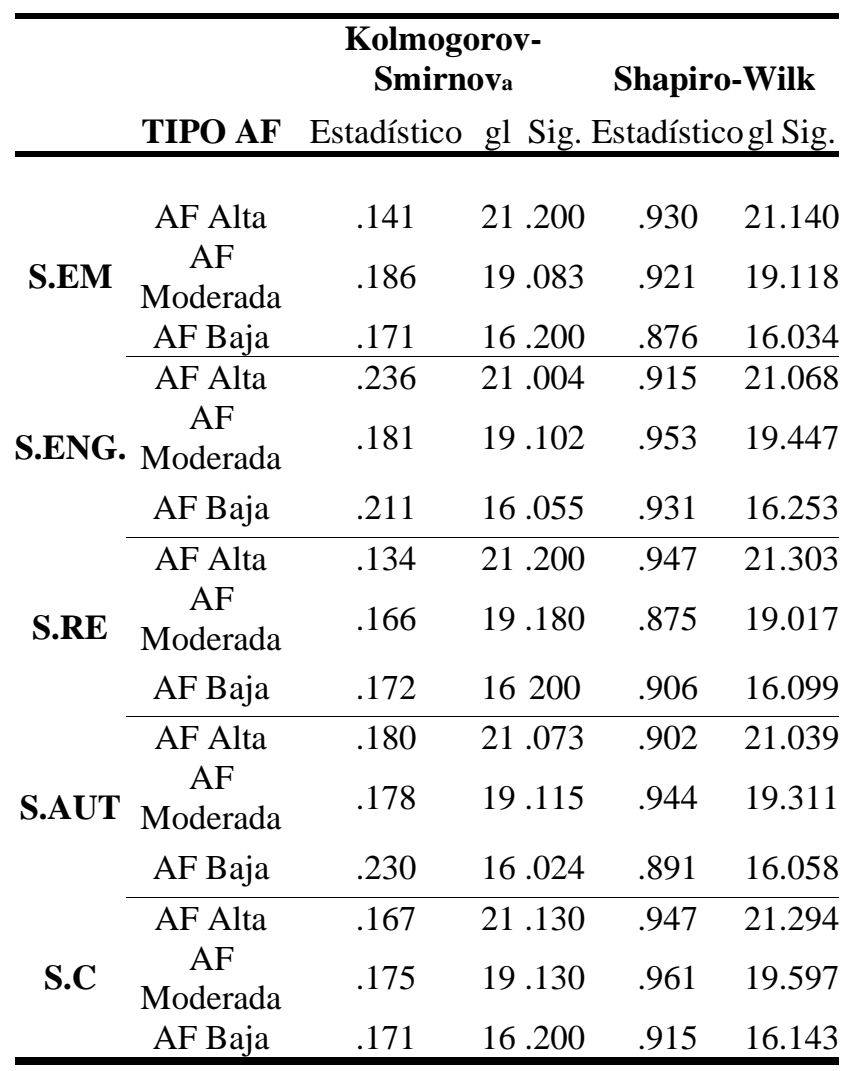

En la Tabla 3 se muestra las medias y desviaciones típicas que existen entre las diferentes variables de los empleados saludables con la práctica de actividad física. Se analizará la media de cada variable con respecto a la cantidad de actividad física organizada en (A) alta, (M) moderada y (B) baja o no práctica de actividad física.

Se harán los cálculos mediante el análisis de la varianza (ANOVA) para las pruebas paramétricas y para las pruebas no paramétricas la ANOVA kruskalwallis entre las medidas de práctica de actividad física y las 5 dimensiones del empleado saludable: emociones positivas (EP), engagement (ENG), resiliencia (RE), autoeficacia (AUT) y competencia (CM).
Los empleados que practicaron actividad física alta y moderada presentan diferencias significativas con respecto a los que practican actividad baja o no práctica en las variables engagement, emociones positivas y resiliencia. Por otro lado, los empleados que practican actividad física alta y moderada presentan índices más altos, pero no significativos que los que practican actividad física baja o no práctica en las variables autoeficacia y competencia.

Tabla 3. Medias empleados saludables y práctica de actividad física.

\begin{tabular}{|c|c|c|c|c|c|}
\hline & TIPO AF & $\mathbf{n}$ & $\mathbf{m}$ & dt & Sig \\
\hline \multirow{4}{*}{ ENG } & $\mathbf{A}$ & 21 & 4.93 & .559 & \multirow{4}{*}{$0.000 * * *$} \\
\hline & $\mathbf{M}$ & 19 & 4.71 & .741 & \\
\hline & B & 16 & 3.29 & .403 & \\
\hline & Total & 56 & 4.39 & .914 & \\
\hline \multirow{4}{*}{$\mathbf{E P}$} & $\mathbf{A}$ & 21 & 4.85 & .823 & \multirow{4}{*}{$0.000 * * *$} \\
\hline & $\mathbf{M}$ & 19 & 4.44 & .810 & \\
\hline & B & 16 & 2.97 & .442 & \\
\hline & Total & 56 & 4.18 & 1.06 & \\
\hline \multirow{4}{*}{$\mathbf{R E}$} & $\mathbf{A}$ & 21 & 4.95 & .731 & \multirow{4}{*}{$0.000 * *$} \\
\hline & $\mathbf{M}$ & 19 & 4.46 & 1.09 & \\
\hline & B & 16 & 3.34 & .674 & \\
\hline & Total & 56 & 4.32 & 1.07 & \\
\hline \multirow{4}{*}{ AUT } & $\mathbf{A}$ & 21 & 5.03 & .773 & \multirow{4}{*}{.247} \\
\hline & $\mathbf{M}$ & 19 & 4.77 & .711 & \\
\hline & B & 16 & 4.62 & .815 & \\
\hline & Total & 56 & 4.82 & .770 & \\
\hline \multirow{4}{*}{$\mathbf{C M}$} & $\mathbf{A}$ & 21 & 4.71 & .778 & \multirow{4}{*}{.093} \\
\hline & $\mathbf{M}$ & 19 & 4.43 & .671 & \\
\hline & B & 16 & 4.15 & .817 & \\
\hline & Total & 56 & 4.46 & .775 & \\
\hline
\end{tabular}

En la Tabla 4 se muestran los resultados de Bonferroni y Games Holmes entre grupos, siendo entre actividad física baja y actividad física alta $p<, 001$, entre la actividad física baja y actividad física moderada $\mathrm{p}$ $<, 001$, y entre actividad física moderada y actividad física alta $p>, 05$, en las tres dimensiones donde existían diferencias significativas. 


\section{Gómez-Chacón, R; Fernández-Martínez, N.}

Tabla 4. Test de Bonferroni.

\begin{tabular}{llll}
\hline & $\begin{array}{l}\text { BAJA } \\
\text { ALTA }\end{array}$ & $\begin{array}{l}\text { BAJA } \\
\text { MODERADA }\end{array}$ & $\begin{array}{l}\text { MODERADA - } \\
\text { ALTA }\end{array}$ \\
\hline EP & $\begin{array}{l}\mathrm{DM}=- \\
1.87 * * *\end{array}$ & $\mathrm{DM}=-1.46^{* * *}$ & $\mathrm{DM}=-.40$ \\
$\mathbf{E N G}$ & $\begin{array}{l}\mathrm{DM}=- \\
1.63 * * *\end{array}$ & $\mathrm{DM}=-1.42 * * *$ & $\mathrm{DM}=-.21$ \\
$\mathbf{R E}$ & $\begin{array}{l}\mathrm{DM}=- \\
1.60 * * *\end{array}$ & $\mathrm{DM}=-1.11 * * *$ & $\mathrm{DM}=.48$ \\
& & \\
\hline $\mathrm{p}<.05, * * \mathrm{p}<.01, * * * \mathrm{p}<.001$ &
\end{tabular}

\section{DISCUSIÓN Y CONCLUSIONES}

Los empleados del centro deportivo-social de la provincia de Sevilla que realizan actividad física moderada y alta presentan un promedio significativamente mayor en emociones positivas, engagement y resiliencia respecto al grupo de actividad física baja o no práctica de actividad física, coincide con los resultados obtenidos por GómezChacón (2017). Además, coincide con los autores Gerber, Jonsdottir, Lindwall y Ahlborg (2014) que afirman que los empleados que realizan actividad física se asocian con perfiles de trabajadores más saludables; y con el estudio llevado a cabo por Chacón-Cuberos, Puertas-Molero y Pérez-Cortés (2017) en el que revelan que practicar actividad física incrementa la capacidad resiliente en estudiantes universitarios. Por otro lado, coincide con los resultados aportados por Boix, León y Serrano (2017) en los que asocian a los empleados que practican pilates niveles más altos en optimismo que los empleados sedentarios. En esta línea Larun, Brurberg y Odgaard-Jensen (2017) subrayan que implementar acciones de actividad física en el lugar de trabajo parece ser una manera eficaz de promover el vigor entre los empleados, que es uno de los tres componentes que definen al engagement (Schaufeli, Salanova, González-Romá y Baker, 2002).

Por otro lado, los empleados que realizan actividad física moderada y alta presentan un promedio mayor, pero no significativo en autoeficacia y competencia respecto al grupo de actividad física baja o no práctica de actividad física, lo cual no coincide con los resultados obtenidos por Gómez-Chacón (2017), que si mostraron diferencias significativas.

\section{Limitaciones.}

Las principales limitaciones de este estudio son los participantes, ya que resulta complejo investigar en determinadas organizaciones y que los gerentes permitan disponer de tiempo para que sus empleados pueden rellenar los cuestionarios. Este hecho reduce la posibilidad de la muestra en determinadas ocasiones como este estudio. Por otro lado, el recabar la información a través de formularios online no se pudieron resolver las dudas que presentarás los trabajadores.

\section{Futuras líneas de investigación.}

Las futuras investigaciones tienen que ir encaminadas a investigar en diferentes empresas, y diferentes sectores, tales como empleados más estáticos en su puesto de trabajo o empleados más dinámicos, siendo empresas más sedentarias o menos.

Por otro lado, la investigación debe ir encaminada a relacionar los empleados saludables, con otras variables tales como el liderazgo, que mejora el engagement y mejora el estado de salud en general (Mazzetti, Vignoli, Petruzziello y Palareti, 2019), la satisfacción laboral que se relaciona positivamente con mayores índices de engagement (Guglielmi, Avanzi, Chiesa, Mariani, Bruni y Depolo, 2019), el emprendimiento, la confianza, potencial creativo.

\section{APLICACIONES PRÁCTICAS}

Los hallazgos encontrados en este estudio indican que, planteando programas de gestión de la salud mediante la práctica de actividad física, bien en la empresa o bien por medio de convenios con centros deportivos, mejorará la resiliencia, las emociones positivas y el engagement repercutiendo en beneficios físicos y psicosociales en los empleados y a su vez repercutirá en las empresas en términos económicos.

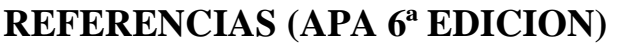

1. Acosta, H., Salanova, M. y Llorens, S. (2011). ¿Cómo Predicen las Prácticas Organizacionales el Engagement en el Trabajo en Equipo?: El Rol de la Confianza Organizacional. Ciencia del Trabajo, 13(41), 125-134. https://doi.org/10.1016/j.rpto.2017.02.005

2. American Psychological Association (2002). Ethical principles of psychologists and code of 


\section{Actividad física y empleados saludables}

conduct. American Psychologist, 57(12), 10601073.

3. Bamford, M., Wong, C., y Laschinger, H. (2013). The influence of authentic leadership and areas of worklife on work engagement of registered nurses. Journal of Nursing Management, 21(3), 529-540. https://doi.org/10.1111/j.13652834.2012.01399.x

4. Blázquez-Manzano, A., León-Mejía, A. y FeuMolina, S. (2015). Intención y práctica de actividad física en maestros españoles. Cuadernos de Psicología del Deporte, 15(2), 163-170.

5. Boix-Vilella, S., León-Zarceño, E. y SerranoRosa, M.A. (2017). Salud psicosocial en trabajadores que practican Pilates: Un estudio descriptivo-comparativo. Ciencia CCD, 12, 2737. https://doi.org/10.12800/ccd.v12i34.829

6. Brown, W.J., Trost, S.G., Bauman, A., Mummery, K. y Owen, N. (2004). Test-retest reliability of four physical activity measures used in population surveys. Journal of Science and Medicine in Sport, $\quad 7(2), \quad 205-215$. https://doi.org/10.1016/s1440-2440(04)80010-0

7. Casimiro, A.J., Artés, E.M., Muyor, J.M y Rodríguez, M.A. (2011). Incidencia de un programa de actividad física en la calidad de vida de los trabajadores en su ámbito laboral. Archivos de medicina del deporte, 23(144), 247-256. https://doi.org/10.17060/ijodaep.2017.n2.v2.1095

8. Chacón-Cuberos, R.; Puertas-Molero, P.; PérezCortés, A. J. (2017). Niveles de resiliencia según práctica de actividad física en estudiantes universitarios de Educación Física. ESHPA Education, Sport, Health and Physical Activity, I(1), 59-67. https://doi.org/10.5672/apunts.20140983.es.(2018/1).131.04

9. Craig, C.L., Marshall, A.L., Sjostrom, M., Bauman, A.E., Booth, M.L., Ainsworth, B.E., Oja, P. (2003). International physical activity questionnaire: 12-country reliabilityand validity. Medicine Science and Sports Exercise, 35(8), 1381-95.

https://doi.org/10.1249/01.mss.0000078924.6145 3.fb

10. De Miguel-Calvo, J.M., Schweiger-Gallo, I., Mozas-Majano, O. y Hernández-López, J.M. (2011). Efecto del ejercicio físico en la productividad laboral y el bienestar. Revista de Psicología del Deporte, 20(2), 589-604. https://doi.org/10.5093/rpadef2018a6
11. Díaz, X., Mena, C.B y Rebolledo A.C. (2012). Propuesta de un programa de promoción de la salud con actividad física en funcionarios públicos. Praxis Educativa, 15(15), 104-109.

12. Flahr, H., Brown, W. J., y Kolbe-Alexander, T. L. (2018). A systematic review of physical activitybased interventions in shift workers. Preventive Medicine Reports, 10, 323-331. https://doi.org/10.1016/j.pmedr.2018.04.004

13. Gerber, M., Jonsdottir, I.H., Lindwall, M., y Ahlborg, G. (2014). Physical activity in employees with differing occupational stress and mental health profiles: A latent profile analysis. Psychology of Sport and Exercise, 15(6), 649-658. https://doi.org/10.1016/j.psychsport.2014.07.012

14. Gómez-Chacón, R., Grimaldi-Puyana, M., Bernal, A. y Fernández-Gavira, J. (2016). La práctica de actividad y su relación con la satisfacción laboral en una organización de alimentación. Journal of Sport Economics \& Management, 6(2), 85-98. https://doi.org/10.6018/sportk.343081

15. Gómez-Chacón, R., Morales-Sánchez, V., Hernández-Mendo, A. y Muñoz-Llerena, A. (2018). La práctica de actividad física y su relación con la satisfacción laboral en una consultora informática. Sport TK: Revista Euroamericana de Ciencias del Deporte, 7(2), 3540. https://doi.org/10.6018/sportk.343081

16. Gómez-Chacón, R. (2017). Análisis del perfil del empleado saludable y su relación con la práctica de actividad física. (Tesis doctoral). Universidad de Sevilla. Sevilla. https://doi.org/10.5585/podium.v6i3.230

17. Gómez-Chacón, R., García-Fernández, J., Morales-Sánchez, V., y Hernández-Mendo, A. (2020). Adaptation and validation of the healthy employee questionnaire of the HERO model. Anales De Psicología / Annals of Psychology, 36(2), 361-369. https://doi.org/10.6018/analesps.395431

18. Guglielmi, D., Avanzi, L., Chiesa, R., Mariani, M.G., Bruni, I. y Depolo, M. (2016). Positive Aging in Demanding Workplaces: The Gain Cycle between Job Satisfaction and Work Engagement. Frontiers in Psychology, 7, 1224. doi: 10.3389/fpsyg.2016.01224

19. Isen, A.M. (2002). Missing in action in the AIM: positive affect's facilitation of cognitive 


\section{Gómez-Chacón, R; Fernández-Martínez, N.}

flexibility, innovation, and problem solving. Psychological Inquiry, 13(1), 57-65.

20. Lisbona, A., Morales, F. y Palaci, F. (2009). El engagement como resultado de la socialización organizacional. International Journal of Psychology and Psychological Therapy, 9(1), 89100.

21. Luthans, F. y Youssef, M. (2004). Human, social and now positive psychological capital management: investing in people for competitive advantage. Organizational Dinamics, 33(2), 143160.

https://doi.org/10.1016/j.orgdyn.2004.01.003

22. Magno Norte da Silva, J., Amaral, L., Marama de Araujo Vieira, E., Karla dos Santos, W., Alver, G., Heuer, V.D.,... Bueno da Silva, L. (2019). A worksite physical activity program and its association with biopsychosocial factors: An intervention study in a footwear factory. Internacional Journal of Industrial Ergonomics, 69 ,

73-79.

https://doi.org/10.1016/j.ergon.2018.10.001

23. Mazzetti, G., Vignoli, M., Petruzziello, G. y Palareti, L. (2019). The Hardier You Are, the Healthier You Become. May Hardiness and Engagement Explain the Relationship Between Leadership and Employees' Health? Frontiers in Psychology, $9, \quad 2784 . \quad$ doi: 10.3389/fpsyg.2018.02784

24. Meneghel, I., Salanova, M. y Martínez, I.M. (2013). El camino de la resiliencia organizacional. Una revisión teórica. Revista de Psicologia, Ciències de l'Educació i de l'Esport, 32(2), 13-24.

25. Ministerio de Sanidad y Consumo. (2005). Estrategia para la nutrición, actividad física y prevención de la obesidad. Recuperado de http://www.aecosan.msssi.gob.es/AECOSAN/we b/nutricion/seccion/estrategia_naos.htm

26. Nelson, D.L. y Simmons, B.L. (2003). Health psychology and work stress: A more positive approach. En J.C. Quick y L. Tetrick (Eds.), Handbook of Occupational Health Psychology (pp. 97-119). Washington, DC: American Psychological Association. https://doi.org/10.1037/10474-005

27. Netz, Y., Wu, M.J., Becker, B.J. y Tenenbaum, G. (2005). Physical activity and psychological wellbeing in advanced age: A meta-analysis of intervention studies. Psychology and Aging,
20(2), 272-284. https://doi.org/10.1037/08827974.20.2.272

28. Palací, F.J. (2009). Psicología de las organizaciones. New York: Pearson Prentince Hall.

29. Pardini, R., Matsudo, S. (2001). Validation of the International Physical Activity Questionaire (IPAQ version 6): pilot study in Brazilian young adults. Revista Brasileira de Medicina do Esporte, 9, 45-51.

30. Salanova, M. (2008). Organizaciones saludables y desarrollo de recursos humanos. Estudios Financieros, 303, 179-214.

31. Salanova, M., Martínez, I.M. y Llorens, S. (2012). Success breeds success, especially when selfefficacy is related with a causality internal attribution. Estudios de Psicología, 33(2), 151165.

https://doi.org/10.1174/021093912800676420

32. Salanova, M. y Schaufeli, W.B. (2009). El engagement en el trabajo. Cuando el trabajo se convierte en pasión. Madrid, España: Alianza Editorial.

https://doi.org/10.5944/rppc.vol.3.num.2.1998.38 60

33. Schaufeli, W.B., Salanova, M., Gonzalez-Roma, V. y Bakker, A.B. (2002). The measurement of engagement and burnout: a two sample confirmatory factor analytic approach. Journal of Happiness Studies, 3(1), 71-92. https://doi.org/10.1023/a:1015630930326

34. Silva, J. M. N. da, Gontijo, L. A., Vieira, E. M. de A., Leite, W. K. dos S., Colaço, G. A., Carvalho, V. D. H. de, ... Silva, L. B. da. (2019). A worksite physical activity program and its association with biopsychosocial factors: An intervention study in a footwear factory. International Journal of Industrial Ergonomics, 69, 73-79. https://doi.org/10.1016/j.ergon.2018.10.001

35. Stanjovik, A.D. (2006). Development of a core confidence-higher order construct. Journal of Applied Psychology, 91(6), 1208-1224. https://doi.org/10.1037/0021-9010.91.6.1208

36. Täuber, S., Mulder, L.B. y Flint, S.W. (2018). The Impact of Workplace Health Promotion Programs Emphasizing Individual Responsibility on Weight Stigma and Discrimination. Frontiers in $\begin{array}{lrrl}\text { Psychology, } & 9, & 2206 . & \text { doi: }\end{array}$ 10.3389/fpsyg.2018.02206 


\section{Actividad física y empleados saludables}

37. Thøgersen-Ntoumani, C., Fox, K.R. y Ntoumanis, N. (2005). Relationships between exercise and three components of mental well-being in corporate employees. Psychology of Sport and Exercise, 6(6), 609-627. https://doi.org/10.1016/j.psychsport.2004.12.004

38. Vera, M., Le Blanc, P.M., Taris, T.W. y Salanova, M. (2014). Patterns of engagement: the relationship between efficacy beliefs and task engagement at the individual versus collective level. Journal of Applied Social Psychology, 44(2),

133-144.

https://doi.org/10.1111/jasp.12219 